\title{
Effect of Vanadium Substitution of Cobalt in $\mathrm{NdCo}_{2} \mathrm{Si}_{2}$
}

\author{
R. Roy ChowdhurY*, S. Dhara And B. BANDYOPADHyay \\ Saha Institute of Nuclear Physics, 1/AF, Bidhannagar, Kolkata 700064, India
}

\begin{abstract}
In $\mathrm{NdCo}_{2} \mathrm{Si}_{2}$, partial substitution of Co by $\mathrm{V}$ having a larger atomic radius results in negative pressure affecting the magnetic properties of the compound. The samples $\mathrm{Nd}\left(\mathrm{Co}_{1-x} \mathrm{~V}_{x}\right)_{2} \mathrm{Si}_{2}(x=0,0.20,0.35)$ have been prepared and characterized using X-ray diffraction. The parent compound $\mathrm{NdCo}_{2} \mathrm{Si}_{2}$ is antiferromagnetic with $T_{\mathrm{N}}$ of $34 \mathrm{~K}$ whereas the temperature dependence of inverse susceptibility in the doped samples show ferrimagnetic behaviour below $45 \mathrm{~K}$. From the magnetic field dependence of magnetization and the coercive fields obtained from hysteresis loops, the doped samples exhibit ferromagnetism below $\approx 20 \mathrm{~K}$. The specific heat studies compare the magnetic transitions in the parent and doped samples. $\mathrm{Nd}\left(\mathrm{Co}_{0.65} \mathrm{~V}_{0.35}\right)_{2} \mathrm{Si}_{2}$ shows a magnetoresistance of $15 \%$ at $4 \mathrm{~K}$.
\end{abstract}

DOI: 10.12693 /APhysPolA.128.530

PACS: $71.20 . \mathrm{Lp}, 75.50 . \mathrm{Gg}, 75.50 . \mathrm{Ee}, 75.50 . \mathrm{Cc}, 75.50 . \mathrm{Dd}$

\section{Introduction}

Intermetallic compounds of the type $\mathrm{RT}_{2} \mathrm{X}_{2}(\mathrm{R}=$ rare earth, $\mathrm{T}=$ transition metal and $\mathrm{X}=\mathrm{Si}$ or $\mathrm{Ge}$ ) crystallize in body centered tetragonal $\mathrm{ThCr}_{2} \mathrm{Si}_{2}$ type structure. The $\mathrm{R}(\mathrm{Nd}), \mathrm{T}(\mathrm{Co})$ and the $\mathrm{X}(\mathrm{Si})$ atoms occupy the $2(\mathrm{a})[0,0,0], 4(\mathrm{~d})\left[0, \frac{1}{2}, \frac{1}{4}\right]$ and $4(\mathrm{e})$ sites $\left[0,0, \mathrm{Z}_{\mathrm{Si}} \approx 0.3742\right]$, respectively, in different layers arranged as $\mathrm{R}-\mathrm{X}-\mathrm{T}-\mathrm{X}-\mathrm{R}$ along $c$-axis. Earlier studies suggest that in these compounds magnetic ordering originates due to the ordering of the $\mathrm{R}^{3+}$ ions with $c$-axis being the easy direction, whereas Co does not carry any moment. In the work reported here non-magnetic Co (atomic radius $1.67 \AA$ ) has been partially replaced by another non-magnetic element $\mathrm{V}$ (atomic radius $1.92 \AA$ ). V substitution causes lattice expansion that can be thought of as a negative pressure. It would be interesting to see how magnetism which is determined by the RKKY interaction in rareearth compounds, is affected by such substitution in these layered materials.

\section{Sample preparation and characterization}

$\mathrm{Nd}\left(\mathrm{Co}_{1-x} \mathrm{~V}_{x}\right)_{2} \mathrm{Si}_{2}(x=0,0.20$, and 0.35$)$ were synthesized by melting the corresponding elements in arc furnace under constant argon flow. The XRD pattern of the samples could be indexed in tetragonal $\mathrm{ThCr}_{2} \mathrm{Si}_{2}$ type structure. The lattice parameter of the parent sample $\mathrm{NdCo}_{2} \mathrm{Si}_{2}$ is in good agreement with the reported data.

TABLE

Lattice parameters of $\mathrm{Nd}\left(\mathrm{Co}_{1-x} \mathrm{~V}_{x}\right)_{2} \mathrm{Si}_{2}$ obtained using Rietveld refinement of XRD data.

\begin{tabular}{c|c|c|c}
\hline \hline $\begin{array}{c}\text { Vanadium } \\
\text { conc. }(x)\end{array}$ & $\begin{array}{c}a, b \\
{[\AA]}\end{array}$ & $\begin{array}{c}c \\
{[\AA]}\end{array}$ & $\begin{array}{c}\text { Unit cell } \\
\text { vol. }\left[\AA^{3}\right]\end{array}$ \\
\hline- & $3.956(2)$ & $9.901(1)$ & $154.95(2)$ \\
0.20 & $3.962(5)$ & $9.954(8)$ & $156.25(2)$ \\
0.35 & $3.963(2)$ & $9.999(9)$ & $157.04(2)$
\end{tabular}

\footnotetext{
* corresponding author; e-mail:
} rajeswari . roychowdhury@saha . ac. in
The Rietveld analysis shows a slight increase in lattice parameter $a$, and a considerable increase in $c$ upon increase in vanadium as shown in Table.

\section{Results and discussion}

3.1. Magnetization measurements

The parent compound $\mathrm{NdCo}_{2} \mathrm{Si}_{2}$ shows antiferromagnetic transition at $34 \mathrm{~K}$ (Fig. 1a). It also exhibits two

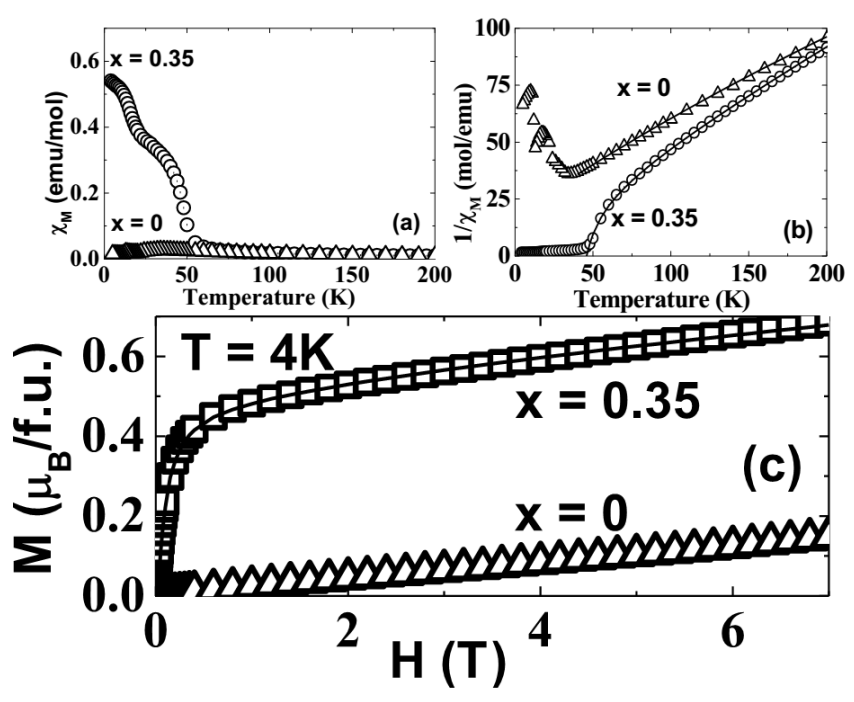

Fig. 1. (a) Temperature dependence of molar magnetic susceptibility $\left(\chi_{M}\right)$ in $\mathrm{Nd}\left(\mathrm{Co}_{1-x} \mathrm{~V}_{x}\right)_{2} \mathrm{Si}_{2},(x=0,0.35)$ at $0.5 \mathrm{~T}$ in between $4-200 \mathrm{~K}$; (b) temperature dependence of inverse molar magnetic susceptibility $\left(1 / \chi_{M}\right)$ in $\mathrm{Nd}\left(\mathrm{Co}_{1-x} \mathrm{~V}_{x}\right)_{2} \mathrm{Si}_{2},(x=0,0.35)$ at $0.5 \mathrm{~T}$ in between 4-200 K; (c) magnetization vs. field data at $4 \mathrm{~K}$ for those two compounds with fitting of Eq. (2).

other transitions at 13 and $24 \mathrm{~K}$ [1] and above $34 \mathrm{~K}$, $\chi_{M}$ follows the Curie-Weiss $(\mathrm{CW})$ behavior. The temperature dependence of inverse molar magnetic susceptibility $\left(1 / \chi_{M}\right)$ in $\mathrm{Nd}\left(\mathrm{Co}_{1-x} \mathrm{~V}_{x}\right)_{2} \mathrm{Si}_{2},(x=0$ and 0.35$)$ at $0.5 \mathrm{~T}$ in between $4-250 \mathrm{~K}$ is shown in Fig. 1b. $\chi_{M}$ in $\mathrm{Nd}\left(\mathrm{Co}_{1-x} \mathrm{~V}_{x}\right)_{2} \mathrm{Si}_{2}$ for $x=0.20$ and 0.35 , follow a ferrimagnetic behaviour, given by [2], 


$$
\chi_{M}=\chi_{0}+\left[(T-\theta) / C+\xi /\left(T-\theta_{1}\right)\right]^{-1}
$$

suggesting involvement of more than one type of magnetic ions in magnetic ordering. The ferrimagnetic ordering temperature thus obtained is $40 \pm 5 \mathrm{~K}$. Above this temperature, the magnetization behaviour is almost paramagnetic. At lower temperatures, $M$ vs. $H$ plots for the substituted samples tend to saturate at $2 \mathrm{~T}$ indicating the appearance of ferromagnetism as a result of $\mathrm{V}$ substitution. At $4 \mathrm{~K}, M$ vs. $H$ for $\mathrm{Nd}\left(\mathrm{Co}_{0.65} \mathrm{~V}_{0.35}\right)_{2} \mathrm{Si}_{2}$ is shown in Fig. 1c. The field dependence of magnetization has been fitted with equation

$$
M=\chi_{0} H+M_{\mathrm{S}} \tan ^{-1}\left(\chi_{1} H\right) .
$$

The first part of the equation gives the paramagnetic contribution to the magnetization, whereas the second term in Eq. (2) represents magnetic field dependence of magnetization in a ferromagnetic material giving saturation of magnetization at high field. At $4 \mathrm{~K}$, the value of $\chi_{0}$ is comparable to the effective moment of $\mathrm{Nd}^{3+}$ as has been obtained from the $C$ values of Eq. (1). The saturation magnetic moment $M_{S}$, increases with $\mathrm{V}$ content and reaches to $\approx 0.35-0.40 \mu_{\mathrm{B}} /$ f.u. in the maximum doped compound. These values are small compared to the free rare-earth ion moments. Interestingly, these values are comparable to magnetic moment of vanadium present in dilute amount in $\mathrm{FeV}$ alloys [3]. Hysteresis behaviour at $4 \mathrm{~K}$ of $\mathrm{Nd}\left(\mathrm{Co}_{0.80} \mathrm{~V}_{0.20}\right)_{2} \mathrm{Si}_{2}$ is shown in Fig. 2 and compared with $M$ vs. $H$ of the antiferromagnetic parent compound. Large hysteresis loops, having high coercivity values $\approx 0.4-0.5 \mathrm{~T}$ have been obtained in doped samples. Temperature dependence of coercivity in $\mathrm{Nd}\left(\mathrm{Co}_{0.65} \mathrm{~V}_{0.35}\right)_{2} \mathrm{Si}_{2}$ has been shown in Fig. 3. In these compounds, the interaction within a layer is ferromagnetic, whereas the nature of the interlayer exchange interaction is antiferromagnetic. With increase in $\mathrm{V}$ substitution, ferromagnetic ordering may appear either in rare earth ions or in Co or $\mathrm{V}$ ions. However, since the paramagnetic part in Eq. (2) appears to be due to Nd ions, there is a possibility that ferromagnetism in $\mathrm{V}$ doped compounds occur in the $\mathrm{Co} / \mathrm{V}$ layer.

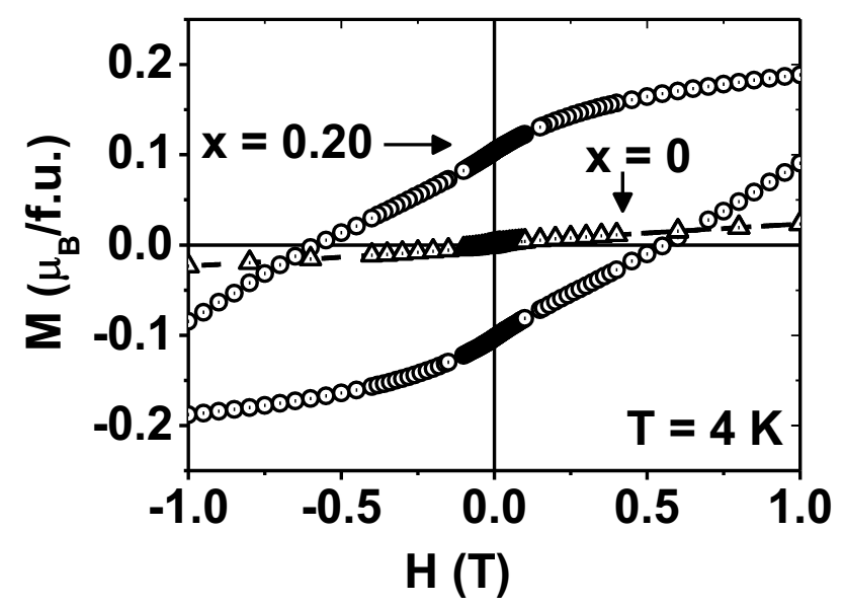

Fig. 2. $\quad M-H$ curve at $4 \mathrm{~K}$ for the parent compound $\mathrm{NdCo}_{2} \mathrm{Si}_{2}$ and the doped sample $\mathrm{Nd}\left(\mathrm{Co}_{0.80} \mathrm{~V}_{0.20}\right)_{2} \mathrm{Si}_{2}$.

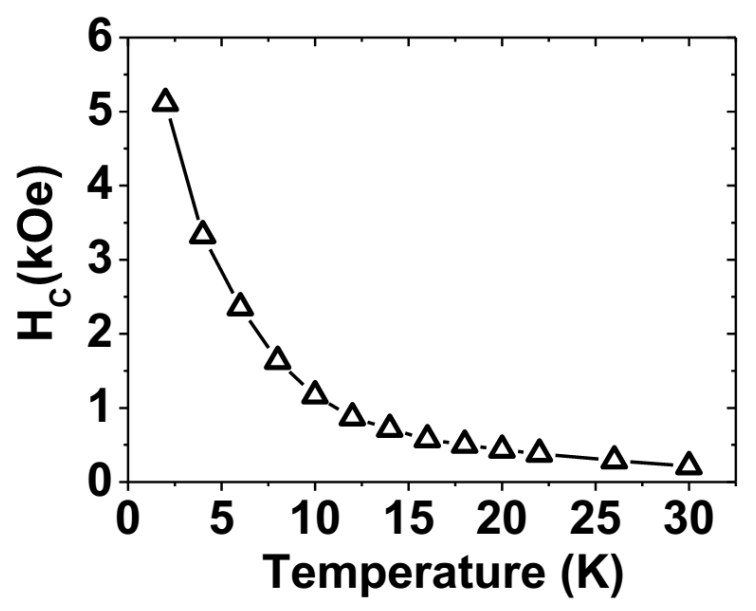

Fig. 3. Temperature dependence of coercive field for $\mathrm{Nd}\left(\mathrm{Co}_{0.65} \mathrm{~V}_{0.35}\right)_{2} \mathrm{Si}_{2}$.

\subsection{Specific heat and resistivity measurements}

For the parent compound the magnetic specific heat measurement shows (Fig. 4) a prominent peak at $T_{\mathrm{N}}=$ $32 \mathrm{~K}$, and also indicates two other transitions due to moment reorientation at $\approx 13$ and $23 \mathrm{~K}$ [1]. The doped compound shows a broad peak at $45 \mathrm{~K}$ which is the position of ferrimagnetic transition as obtained from $\chi_{M}$ vs. $T$ (Fig. 1a and b), and a sharp peak at $26 \mathrm{~K}$ below which ferromagnetic behaviour is obtained. The lattice contribution to the specific heat is estimated from the measurement of specific heat of isostructural nonmagnetic compound $\mathrm{LaCo}_{2} \mathrm{Si}_{2}$. The renormalization of the Debye temperature due to different molar masses between La and Nd was carried out following the Debye method. The Debye temperature values obtained are $\theta_{\mathrm{D}} \approx 427$, 440 , and $510 \mathrm{~K}$ for $x=0,0.20$, and 0.35 , respectively.

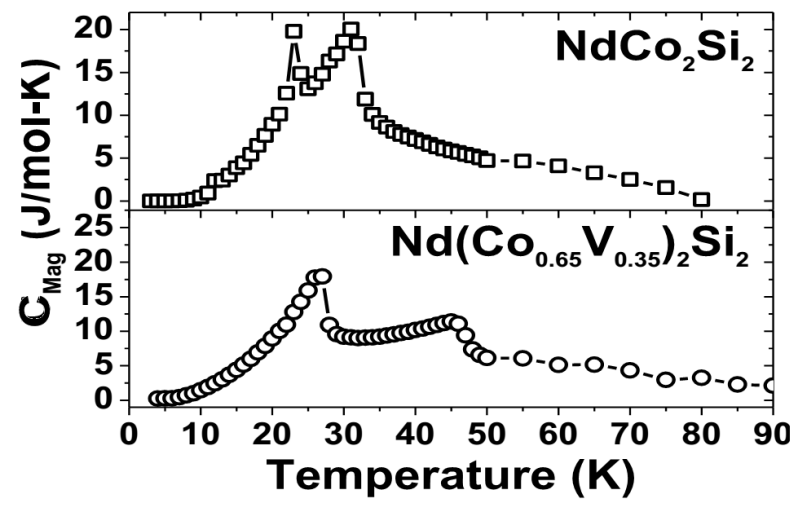

Fig. 4. Temperature dependence of magnetic contribution to specific heat $\left(C_{\mathrm{mag}}\right)$ for parent compound and doped sample $\mathrm{Nd}\left(\mathrm{Co}_{0.65} \mathrm{~V}_{0.35}\right)_{2} \mathrm{Si}_{2}$.

The parent compound $\mathrm{NdCo}_{2} \mathrm{Si}_{2}$ has a positive magnetoresistance (MR). The doped sample, e.g., $\mathrm{Nd}\left(\mathrm{Co}_{0.65} \mathrm{~V}_{0.35}\right)_{2} \mathrm{Si}_{2}$ is ferromagnetic and shows small negative MR down to about $10 \mathrm{~K}$. Below $8 \mathrm{~K}$, MR becomes positive and goes to $15 \%$ at $4 \mathrm{~K}$ and $9 \mathrm{~T}$ 


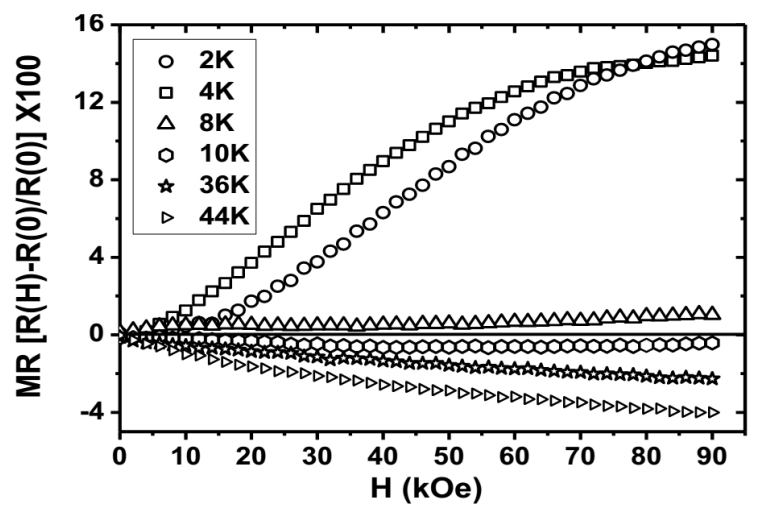

Fig. 5. Magnetoresistance (MR) vs. field at different temperatures for $\mathrm{Nd}\left(\mathrm{Co}_{0.65} \mathrm{~V}_{0.35}\right)_{2} \mathrm{Si}_{2}$.

(Fig. 5). For a ferromagnetically ordered bulk polycrystalline material one usually expects a negative sign of MR. However, similar anomalous MR behaviour have been observed earlier in isostructural ferromagnetic $\mathrm{LaMn}_{2} \mathrm{Si}_{2}$ [4]. This positive MR may arise in presence of field induced pseudogaps in some portions of the Fermi surface.

\section{Conclusions}

The parent compound, $\mathrm{NdCo}_{2} \mathrm{Si}_{2}$ is antiferromagnetic, in which Co does not carry magnetic moment. On V sub- stitution, the doped samples show ferrimagnetic behaviour below $\approx 40 \pm 5 \mathrm{~K}$, whereas ferromagnetism is observed below $\approx 18 \mathrm{~K}$ with the $M$ vs. $H$ curves showing a tendency of saturation and having a hysteresis loop. In these layered compounds, the dominant AFM interaction is along $c$-axis whereas in $a b$ plane there exists a ferromagnetic interaction. There is a possibility of reduction of AFM interaction, and simultaneously FM interaction may be favoured due to the considerable lattice expansion along $c$-axis caused by vanadium substitution (which may lead to the localization of $d$ electrons). Magnetoresistance in the ferromagnetic sample shows a positive value at low temperatures which may be due to opening of pseudogaps at the Fermi surface. Detailed investigation and microscopic studies of these alloys to identify the local moment, whether $\mathrm{Co}$ or $\mathrm{V}$ would be necessary to understand magnetism in these multilayered compounds.

\section{References}

[1] T. Shigeoka, N. Iwata, Y. Hashimoto, Y. Andoh, H. Fujii, J. Phys. (France) 49, C8-431 (1988).

[2] J.S. Smart, Am. J. Phys. 23, 356 (1955).

[3] I. Mirebeau, G. Parette, J. Appl. Phys. 53, 1960 (1982).

[4] R. Mallik, E.V. Sampathkumaran, P.L. Paulose, Appl. Phys. Lett. 71, 2385 (1997). 